\title{
Fully consistent density functional theory determination of the insulator-metal transition boundary in warm dense hydrogen
}

\author{
Joshua Hinz, ${ }^{1}$ Valentin V. Karasiev,,${ }^{1, *}$ S. X. Hu $\odot,{ }^{1}$ Mohamed Zaghoo, ${ }^{1}$ Daniel Mejía-Rodríguez $\odot,{ }^{2}$ \\ S. B. Trickey $\odot,^{2}$ and L. Calderín ${ }^{3}$ \\ ${ }^{1}$ Laboratory for Laser Energetics, University of Rochester, Rochester, New York 14623, USA \\ ${ }^{2}$ Quantum Theory Project, Department of Physics, University of Florida, Gainesville, Florida 32611, USA \\ ${ }^{3}$ Department of Materials Science and Engineering, University of Arizona, Tucson, Arizona 85721, USA
}

(Received 7 February 2020; revised 11 May 2020; accepted 20 August 2020; published 10 September 2020)

\begin{abstract}
Using conceptually and procedurally consistent density functional theory (DFT) calculations with an advanced meta-generalized gradient approximation (GGA) exchange-correlation functional in ab initio Born-Oppenheimer molecular dynamics (BOMD) simulations, we determine the insulator-metal transition (IMT) of warm dense fluid hydrogen from 50 to $300 \mathrm{GPa}$ to be in better agreement with experiment than previous DFT predictions. The inclusion of nuclear quantum effects via path-integral molecular dynamics (PIMD) sharpens the transition and lowers its temperature relative to the BOMD results. A rapid decrease in the molecular character of the system, as observed via the ionic pair correlation function, coincides with an abrupt conductivity increase, confirming a metallic transition due to molecular hydrogen dissociation that is coincident with abrupt band-gap closure. Comparison of the PIMD and BOMD results clearly demonstrates an isotope effect on the IMT. Exploitation of differing methodologies for using the orbital-dependent and deorbitalized versions of the meta-GGA enables us to quantify exchange-correlation approximation effects. Distinct from stochastic simulations, these results do not depend upon any clever but uncontrolled combination of ground-state and finite- $T$ methodologies and should provide a reliable benchmark for further studies.
\end{abstract}

DOI: 10.1103/PhysRevResearch.2.032065

\section{INTRODUCTION}

\section{A. Motivation}

The liquid-liquid insulator-to-metal transition (IMT) of warm dense hydrogen isotopes is a fundamental problem of great interest for a variety of scientific disciplines. For planetary science, accurate determination of the IMT is critical for modeling the interior dynamics and evolution of Jovianlike planets [1] due to the abundance of $\mathrm{H}$ in the universe. The metallization of $\mathrm{H}$ is believed to be essential for $\mathrm{H}-\mathrm{He}$ demixing, a central feature in internal structure models that causes a clear boundary between He-rich and He-poor layers. That boundary is paramount to understanding the dynamo and the zonal flow depth within Jovian-like planets [2]. Interest in the metallization of fluid $\mathrm{H}$, again in the context of planetary science, has been heightened in recent years due in part to the measurements of Jupiter's magnetic field by the Juno mission [3]. An essential prerequisite for quantitative modeling of all such planetary phenomena is an accurate equation of state (EOS) that includes the IMT correctly [4]. Furthermore, and independently of planetary physics, an accurate EOS for $\mathrm{H}$

\footnotetext{
*vkarasev@lle.rochester.edu

Published by the American Physical Society under the terms of the Creative Commons Attribution 4.0 International license. Further distribution of this work must maintain attribution to the author(s) and the published article's title, journal citation, and DOI.
}

and its isotopes also is essential for progress in inertial confinement fusion and high-energy-density physics research [5].

Despite its importance, accurate determination of the IMT boundary remains a long-standing challenge. Here, we make a major step forward on the theory/computation side by providing a single, conceptually consistent density functional theory (DFT) treatment, with the best-balanced modern approximate exchange-correlation (XC) functional available. Our results, with and without nuclear quantum effects (NQEs), are mostly consistent with experimental findings and confirm the best previously available results from ingenious but ad hoc combinations of DFT and stochastic methods.

\section{B. Experimental background}

Of the previous experiments, gas-gun work by Weir et al. [6] measured deuterium (D) resistivity but not directly the temperature to place an IMT point at $140 \mathrm{GPa}$ with an estimated temperature $T_{m}>2500 \mathrm{~K}$. Static compression experiments (SCEs) on $\mathrm{H}$ [7-9] determined a set of IMT boundary points, as interpreted by plateaus in the laser heating curve, to occur at pressures $P_{m}<180 \mathrm{GPa}$ and $T_{m}$ significantly below the Weir et al. value. Optical measurements in Ref. [8] indicated that the laser heating curve plateaus immediately precede a substantial reflectivity increase. This coincidence suggests that such plateaus are evidence of the latent heat of a first-order transition. However, McWilliam and co-workers [10] later determined that the plateaus are not from 
the IMT latent heat but from the onset of $\mathrm{H}$ absorbance at the experimental optical frequencies [7-9].

Follow-up SCEs by Zaghoo and Silvera [11] refined the measured reflectance of hydrogen, resulting in an upward shift of the IMT boundary by $\approx 500 \mathrm{~K}$ compared to previous SCEs, yet significantly below the Weir et al. results. Subsequent SCEs by Zaghoo [12] on D at the same pressures as the previous $\mathrm{H}$ measurements [9] gave an IMT boundary $\approx 600 \mathrm{~K}$ higher than for $\mathrm{H}$. Identification as an isotope effect was strengthened by the fact that the temperature shift is on the order of the $\mathrm{H}_{2}-\mathrm{D}_{2}$ bond energy difference.

The IMT boundary location was clouded further, however, by the dynamic compression experiments (DCEs) of Knudson et al. [13] on D which put the IMT at unprecedented pressures, $P_{m}>300 \mathrm{GPa}$, with a weak $T$ dependence. A reanalysis of the Ref. [13] data by Celliers (see the Supplemental Material of Ref. [14]) reduced the estimate to $T_{m}<1000 \mathrm{~K}$. That reanalysis, in conjunction with the DCE results of Celliers [14], forms a clear IMT boundary for D and further supports the notion of differing boundaries for $\mathrm{H}$ and its isotopes; see the present state of affairs as displayed in Fig. 1. However, debate remains on the experimental side concerning Celliers' reanalysis of the Knudson et al. DCE results [15,16]. See also Desjarlais et al. [17].

\section{Theoretical background}

Theoretical predictions of the IMT boundary have been broadly inconsistent, agreeing at best, with some of the experimental data. The obvious causal suspect is the diversity of approximations used. DFT, the most-used tool, in principle gives the exact solution to the quantum many-body electronic problem. However, the XC free-energy functional must be approximated $[18,19]$. Some of the earlier (and a few more recent) H IMT predictions from DFT were done in the Born-Oppenheimer (BO) approximation [20-25] and employed the ground-state Perdew-Burke-Ernzerhof (PBE) generalized gradient approximation (GGA) XC functional [26]. Those results demonstrated no more than $\approx 25 \mathrm{GPa}$ pressure difference from earlier SCEs [7-9], an IMT phase boundary in the lower-pressure liquid regime. (Note that the use of a ground-state XC functional with Fermi-Dirac finite- $T$ occupations instead of a full free-energy functional is well justified for the temperatures of interest $[27,28]$.) With the addition of NQEs [29], the IMT predicted by PBE shifts toward lower pressures, away from the experimental data, for example, by more than $50 \mathrm{GPa}$ below the transition observed in Ref. [11]. The incorporation of van der Waals ( $v d W)$ interactions [13], via the vdW-DF1 [30] and vdW-DF2 [31] XC functionals, shifted the predicted IMT boundary substantially up, by 100 and $200 \mathrm{GPa}$, respectively. Recently, Geng et al. assumed pragmatically that the IMT boundary lies between the values from the PBE and vdW-DF1 XC functionals [23]. Though plausible, the separation is uncontrolled and large, especially for vdW-DF2 vs PBE, once NQEs are included; see Ref. [32] and references therein. They showed that the revised Vydrov-van Voorhis nonlocal correlation (rVV10) functional combined with the PBE0 hybrid functional seemed to work best, presumably from superior treatment of $\mathrm{H}_{2}$ dissociation.

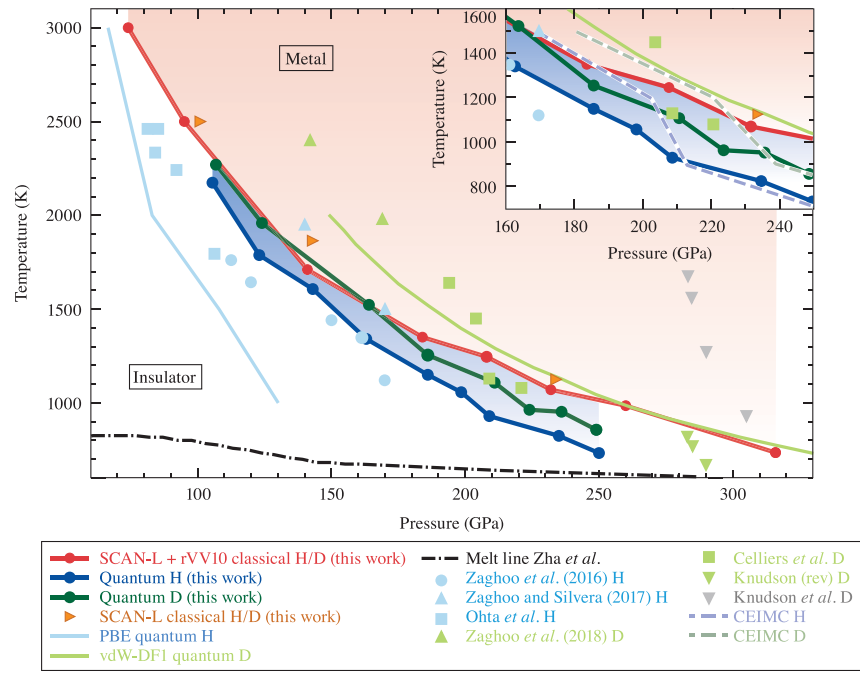

FIG. 1. Emerging picture of the hydrogen and deuterium insulator-to-metal transition (IMT). Blue symbols show experimental results for hydrogen $[7,8,10]$. Upright blue triangles are the IMT from measured reflectivities [10]. All other blue symbols are from the laser heating curve plateau. Green symbols show reflectivity results for deuterium [12-14]. The black dashed and dotted curve is the melt line based on Ref. [61]. The solid light blue and solid light green curves are the predictions of PBE [29] and vdW-DF1 [13], respectively, for the case of quantum nuclei. Within this work all IMT temperature points are determined when the system reaches $2000 \mathrm{~S} / \mathrm{cm}$, which is indicated with solid circles or right pointed triangles. The corresponding solid lines between the solid circles are a guide to the eye. The red curve with circles is the SCAN-L+rVV10 prediction with classical nuclei and the orange triangles pointed to the right are three of the same classical nuclei predictions without the rVV10 correction. The corresponding blue and green curves with circles are the predictions with NQEs for hydrogen and deuterium, respectively. The red-shaded region indicates where classical or quantum $\mathrm{H}$ is metallic, whereas the blue-shaded region indicates where only quantum $\mathrm{H}$ is metallic. Inset: With NQE inclusion an apparent step in the IMT boundary appears. This feature apparently has not been seen previously in DFT studies but appears to be in agreement with CEIMC results which are indicated by the dashed blue (hydrogen) and dashed green (deuterium) curves [33]. Further analysis is required to ascertain the underlying cause of that feature.

Hybrid functionals, however, introduce another uncontrolled approximation, the amount of single-determinant exchange.

Hydrogen IMT predictions $[33,34]$ also have come from coupled electron-ion Monte Carlo (CEIMC) calculations [35] and other quantum Monte Carlo (QMC) methods [1]. In principle, CEIMC can treat the electrons and ions on the same footing. CEIMC calculations in practice, however, are a combination of methods. Ground-state QMC (not finite $T$ ) is used to generate the electronic forces that drive the ions in the BO approximation [35]. Ionic position snapshots then are used to generate Kohn-Sham (KS) orbitals and eigenvalues from the Heyd-Scuseria-Ernzerhof (HSE06) range-separated hybrid XC [36] functional. Those in turn are used to calculate Kubo-Greenwood (KG) conductivities [37,38]. The result is physically inconsistent: The ground-state QMC electron density that drives the ions has no systematic relationship 
to the finite- $T$ electron density that determines the electrical conductivity. Additionally, the electronic QMC calculations start from another set of KS orbitals. Different XC choices for generating them can shift the calculated IMT by as much as $25 \mathrm{GPa}$ (see Supplemental Material in Ref. [33]). The effect of using HSE-derived orbitals has not been assessed. (Use of HSE06 is arbitrary, albeit defensible. Screened short-range single-determinant contributions give spectra that mimic the proper self-energy operator results.) With a disjoint collection of methods comprising CEIMC in practice, it is important that the results be validated by an internally consistent methodology, particularly since predictions based on alternative QMC methodology predict a boundary more than $50 \mathrm{GPa}$ lower [1].

The unanswered question is this: What IMT emerges from a straightforward, internally consistent DFT calculation with a modern meta-GGA functional? The meta-GGA aspect is crucial. Lower-rung functionals are intrinsically incapable of balanced handling of molecular dissociation and solid effects.

\section{METHODS AND PROCEDURES}

We answer with just such calculations of the $H$ and D IMT boundaries. We use the most refined meta-GGA extant, the strongly constrained and appropriately normed (SCAN) functional [39]. It is built to satisfy all 17 constraints that a meta-GGA can satisfy and is normalized to be exact or nearly exact on systems for which an approximate $\mathrm{XC}$ functional can be. It has been demonstrated to perform well on both molecules and solids and to combine well with the rVV10 [40,41] long-range vdW functional [42]. With SCAN+rVV10, we provide a complete, consistent DFT treatment of the IMT boundary by delineating NQEs via a comparison of $a b$ initio path-integral molecular dynamics (quantum nuclei, PIMD) and BO molecular dynamics (classical point nuclei, BOMD) calculations and the determination of band-gap effects by a comparison of generalized KohnSham (gKS) and ordinary KS solutions.

BOMD calculations were done with the Vienna ab initio simulation package (VASP) [43-45]. All simulations used 256 atoms in a cubic supercell, with lattice constants ranging from 7.05 to $10.27 \AA$, at the $\Gamma$ point with 168 bands. The simulations were spin unpolarized. The bare Coulomb ionic potential was handled with the projector augmented-wave (PAW) [46] method. (Note: These are PBE PAWs, as with all VASP PAW calculations with SCAN.) Initial convergence tests were performed at $0.6 \mathrm{~g} / \mathrm{cm}^{3}$ and $2500 \mathrm{~K}$ to ensure the energy and pressure converged within $1 \%$ and $2.5 \%$, respectively (subsequent checks along an entire isochore point to a pressure error $\leqslant 5 \%$ ), with subsequent checks performed throughout the study. Ring-polymer PIMD [47] calculations used the i-PI interface [48,49] with QUANTUM ESPRESSO [50,51] and eight beads. All PIMD and BOMD simulation parameters were consistent. However, the PIMD calculations used a local pseudopotential [52]. Importantly, we did both H and D PIMD simulations, not done previously in DFT or CEIMC.

Total simulation costs from a meta-GGA XC functional can be formidable. Therefore, most of the BOMD simulations used the deorbitalized version of SCAN, SCAN-L $[53,54]$. However, the majority of the PIMD simulations used SCAN because SCAN-L was unavailable in QUANTUM ESPRESSO at the time. In both, the rVV10 correction was used with the same parameters. From 20 snapshots for each trajectory, we did $\mathrm{KG}$ calculations of the dc conductivity, extracted in the static field limit, and of the reflectivity, as well as an analysis of the ionic pair correlation functions (PCFs) and band gaps (structural properties are calculated over the last 4000 steps of the MD run). For these, the KG calculations used KS eigenvalues and orbitals from SCAN-L+rVV10.

Several subsidiary calculations confirm the limited, inconsequential effects of SCAN versus SCAN-L. KG dc conductivity calculations were done with both SCAN+rVV10 and SCAN-L+rVV10 orbitals and eigenvalues on a single set of PIMD SCAN+rVV10 ion configurations. Note that the SCAN+rVV10 KG calculations are performed in QE (see Ref. [55] for details), while the SCAN-L+rVV10 KG calculations were from VASP. Similarly, differences in ion configurations from SCAN versus SCAN-L were assessed by BOMD calculations along the 0.8 and $1.0 \mathrm{~g} / \mathrm{cm}^{3}$ isochores. Details and a complete list of simulation details are provided in the Supplemental Material [56].

To follow a thermodynamic path as consistent as possible with the SCEs [7-12], the MD simulations were done along sets of isochores (except for the two lowest-pressure transition points along the 2500 and $3000 \mathrm{~K}$ isotherms) with densities from 0.8 to $1.15 \mathrm{~g} / \mathrm{cm}^{3}$. Consistent with the literature $[6,13,14]$, a minimum dc conductivity of $2000 \mathrm{~S} / \mathrm{cm}$ was the primary criterion for determining the metallic transition temperature (see the vertical lines of Fig. 2). That yields the solid curves with symbols and labeled "this work" in Fig. 1 (see the discussion of this threshold in Ref. [11]). We note that use of $\Gamma$ point sampling for BOMD simulations along the $0.95 \mathrm{~g} / \mathrm{cm}^{3}$ isochore results in a metallization pressure with a $5 \%$ upward shift as compared to various other Brillouin zone samplings [56]. More importantly, the resulting metallic transition temperature is relatively unaffected as the shift is on the order of two to three error bars in the calculated IMT temperature. However, the abruptness of the slope change in pressure along the isochore is diminished noticeably by use of the $\Gamma$ point, with the result that we can locate the transition but not characterize the transition type unequivocally (a task for future work).

\section{RESULTS}

Figure 1 shows the essential consequences of NQE inclusion in a consistent DFT framework. At any given $P$, the IMT for quantum $\mathrm{D}$ is above that for quantum $\mathrm{H}$. This matches experimental findings at least semiquantitatively. The H IMT is closer to experiment, however, than for D. Without NQEs, the calculated IMT lies higher in $P$ and $T$. Unsurprisingly, as it incorporates less physics, the BOMD IMT agrees less well with experiment.

Detailed analysis begins with Fig. 2(a). It shows the dc conductivity for $\mathrm{H}$ at 0.9 and $1.0 \mathrm{~g} / \mathrm{cm}^{3}$ from both PIMD with SCAN ionic configurations and BOMD with SCAN-L configurations (both with rVV10). Measured against the 2000 $\mathrm{S} / \mathrm{cm}$ criterion, there are dc conductivity increases of four orders of magnitude both with and without NQEs. Consistent with the Zaghoo and Silvera measurements [11] and with an IMT, the corresponding reflectivity [Fig. 2(b)] at $\hbar \omega \approx$ 

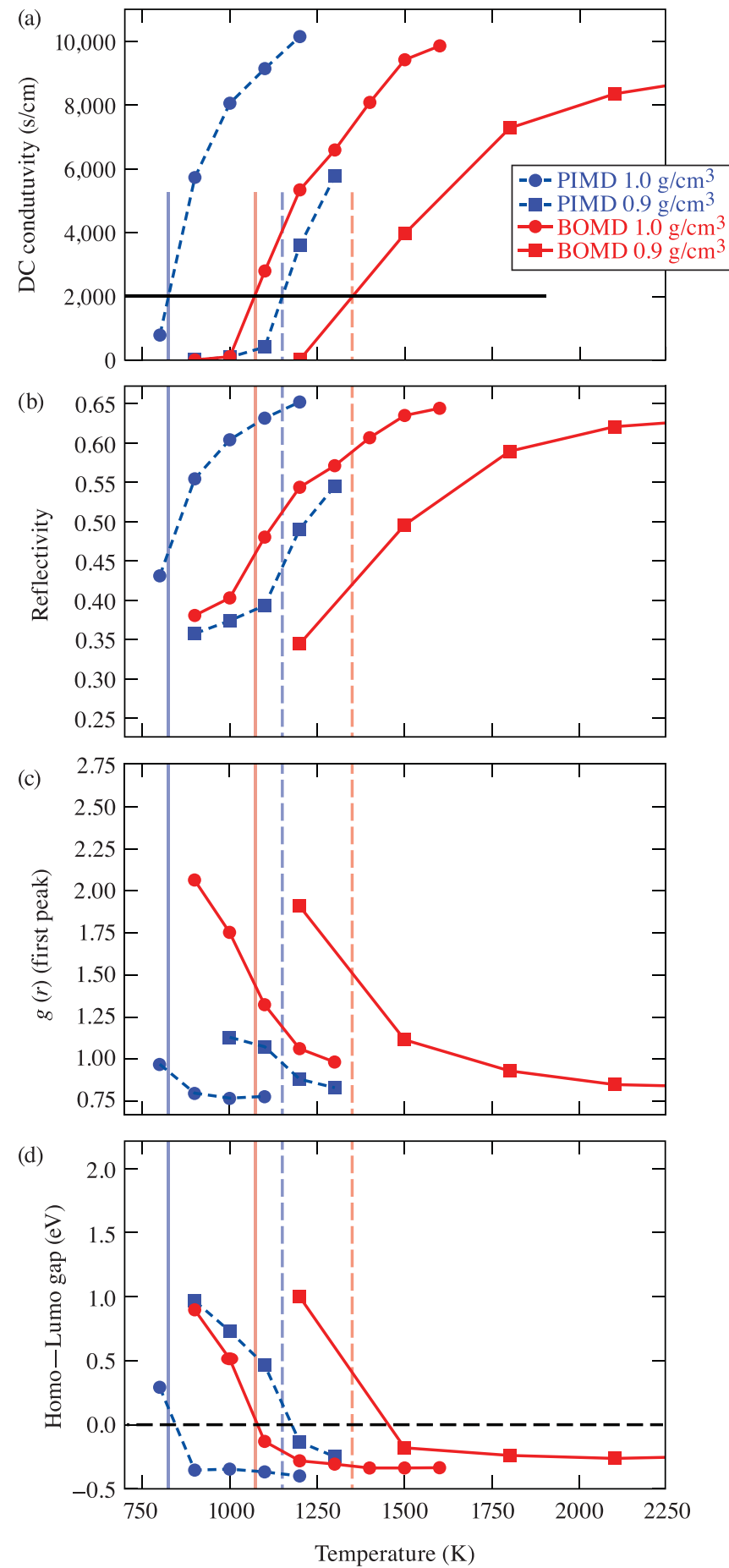

FIG. 2. (a) Calculated dc conductivity of hydrogen along the 0.9 (squares) and $1.0 \mathrm{~g} / \mathrm{cm}^{3}$ (circles) isochores for quantum (blue) and classical (red) ions. The black horizontal line indicates the primary IMT location criterion: metallic de conductivity $>2000 \mathrm{~S} / \mathrm{cm}$. Vertical lines are guides to the eye to mark the temperature at which $2000 \mathrm{~S} / \mathrm{cm}$ is crossed. (b) Corresponding reflectivity at $\sim 1.96 \mathrm{eV}$ relative to vacuum. (c) Height of the first peak of the ionic PCF, a measure of the molecular character of the system. (d) Indirect energy band gap [identified from the $0 \mathrm{~K}$ highest occupied molecular orbital (HOMO)-lowest unoccupied molecular orbital (LUMO) gap; see text]. The horizontal dotted black line indicates gap closure. Negative values indicate that the LUMO minimum lies below the HOMO maximum.
$1.96 \mathrm{eV}$ (calculated relative to vacuum) tends to have an abrupt slope change (see Supplemental Material [56]) that coincides with the dc conductivity increase. However, unlike the observed reflectivity saturation at 0.55 [11,12], Fig. 2(b) indicates possible saturation around 0.65 . The feature appears to be dependent on the system state conditions [56].

The ionic PCF provides an upper bound on the number of possible molecules [57] and gives molecular bond length information. In this context, one sees the improvement provided by an XC functional consistent for both molecular and atomic systems. It is known that PBE underestimates the metallization pressure while vdW-DF1 overbinds $\mathrm{H}_{2}$ molecules and hence delays dissociation (see Ref. [23]). Due to the sensitivity of the IMT to the underlying sampling of the ion configuration space, a more accurate $\mathrm{XC}$ functional for this system should provide structural properties that lie within those bounds. A comparison of the PCF for classical ions [56] demonstrates that both the molecular character and average bond length predicted by SCAN-L+rVV10 for this system do in fact lie between those from PBE and vdW-DF1. It thus is plausible that the success of SCAN at predicting structural properties of various other systems $[39,42,53,54]$ will translate to a better description of the dissociation process of fluid hydrogen and hence an improved IMT boundary compared to results of simpler functionals.

Analysis of the PCF [Fig. 2(c)] indicates a rapid change in the molecular character of the system at the metallic transition onset. NQE inclusion dramatically enhances $\mathrm{H}_{2}$ dissociation prior to the onset of metallic character. This is indicated by a clear slope change, compared to the classical ion case, in the height of the first PCF peak at the $2000 \mathrm{~S} / \mathrm{cm}$ criterion. That increase in the cumulative dissociation amount shifts the IMT towards lower $T$ (recall Fig. 1) and produces a steeper slope in both the dc conductivity and reflectivity as compared to the classical ion case. Further confirmation of $\mathrm{H}_{2}$ dissociation can be seen from the vibration/rotational spectrum of the system obtained from the real part of the Fourier transform of the velocity autocorrelation function. At metallization, abrupt changes are observed in the height of all three visible peaks of the spectrum (PIMD case) [56]. The changes further confirm depletion of the molecular character of the system at the IMT. This connection between dissociation and metallization recently has been demonstrated experimentally [58] at pressures and temperatures just outside the range investigated here. Inclusion of NQEs also induces a clear splitting of the IMT boundaries for $\mathrm{H}$ and D (again, see Fig. 1). That is an unambiguous isotope effect; only the ionic mass differs between the two simulations. Although apparent, the splitting is $\approx 300$ $\mathrm{K}$ smaller than what is observed experimentally $[11,12]$. The origin of that discrepancy is not obvious.

Figure 2(d) is an analysis of the indirect energy gap of the system, defined in terms of the highest occupied and lowest unoccupied $\mathrm{KS}$ bands at $T=0 \mathrm{~K}$ (HOMO, LUMO respectively). They can be identified by symmetry at any $T$. (Observe that the HOMO-LUMO gap defined thus is a good approximation for the finite- $T$ system of interest here because the relevant temperatures never exceed $2 \%$ of the electron Fermi temperature. This is the same reason that the ground-state approximation is valid for the $\mathrm{XC}$ free energy; recall above.) The bands in this case are from SCAN-L (again snapshots are 
taken and calculations performed but with a denser $k$ mesh). In both the classical and quantum ion cases, a clear energy gap closure is seen within $100 \mathrm{~K}$ of the minimum metallic dc conductivity onset. That closure further justifies the use of $2000 \mathrm{~S} / \mathrm{cm}$ as the criterion marking the metallic transition onset. Together with the PCF analysis, it also provides a strong basis for asserting that the metallic transition of fluid hydrogen is driven by abrupt band-gap closure from the dissociation of insulating $\mathrm{H}_{2}$ into metallic atomic hydrogen.

\section{DISCUSSION}

Several features of the calculated transition boundary (Fig. 1) are of interest. First, with NQEs that boundary agrees well with the experimental data for $\mathrm{H}$, lying as it does between the absorption onset [7-9,11] and the reflectivity increase [11]. This feature is important, because conventional semilocal functionals, including SCAN-L, underestimate the insulating band gap [59]. That could lead to overestimated conductivity and underestimation of the IMT temperature. We assessed the magnitude of that effect in two ways. First, we did KG calculations of the dc conductivity with both SCAN+rVV10 and SCAN-L+rVV10 on the same set of ion configurations (from the PIMD SCAN+rVV10 trajectories). The average difference between the two IMT boundaries (again with the $2000 \mathrm{~S} / \mathrm{cm}$ criterion) is $11 \mathrm{~K}$. The difference never exceeds $20 \mathrm{~K}$. In contrast, the shift in the metallic transition temperature associated with the standard error on the dc conductivity from the set of snapshots is $\pm 30 \mathrm{~K}$. Second, as a probe of band-gap underestimation, we calculated the band gap with the HSE06 [60] XC functional. At fixed $T$, such an underestimation leads to overestimated conductivities, thus underestimated $T_{m}$ from those conductivities. The HSE06 gap is, as expected, larger than the SCAN-L+rVV10 value, but the HSE06 gap closure occurs within $50 \mathrm{~K}$ of that from SCAN$\mathrm{L}+\mathrm{rVV} 10$. That difference is on the order of the error bar in the IMT temperature. Thus, the residual self-interaction error in SCAN-L+rVV10 does not alter the temperature-dependent KS spectrum enough to affect the metallization state point for the underlying ion configuration space sample. The use of the $2000 \mathrm{~S} / \mathrm{cm}$ criterion is validated by the finding that the $\mathrm{dc}$ conductivity increases by four or more orders of magnitude at the onset of metallization for a wide range of thermodynamic conditions. At the scale of that criterion, band-gap underestimation effects are washed out. As the dc conductivity change becomes more gradual, presumably because of the existence of a critical point, the use of a specific dc conductivity threshold becomes less reliable. Thus the $2000 \mathrm{~S} / \mathrm{cm}$ threshold and band-gap closure for a particular XC functional may decouple in terms of the predicted temperature. Combinations of PBE and SCAN-L+rVV10 in the MD and KG calculations confirm this notion [56], although the discrepancy is still on the order of a few temperature error bars. Other combinations might have substantially larger shifts, an illustration of the effect of inconsistencies.

Here and in previous studies [4,13,19-21,54], it is found that the underlying set of ion configurations at each set of thermodynamic conditions has the strongest influence on the resulting IMT. Thus, it is important to know the magnitude of effects from trajectory differences between SCAN+rVV10 and SCAN-L+rVV10. To assess that, we compare the dc conductivity from KG with SCAN-L+rVV10 based on BOMD snapshots with either SCAN-L+rVV10 or SCAN+rVV10. The 0.8 and $1.0 \mathrm{~g} / \mathrm{cm}^{3}$ isochores were chosen as representative. On both isochores the SCAN to SCAN-L shift is 5\% of the IMT temperature. Again, see the Supplemental Material for further details [56].

Third, the inclusion of the long-range vdW interactions with the rVV10 correction appears to have the largest impact on the IMT boundary between 100 and $200 \mathrm{GPa}$. Note that in this study the rVV10 correction was implemented so as to describe only the long-range interactions while preserving the short- and intermediate-range description of SCAN-L (see Ref. [42]). That differs from the use of rVV10 in Ref. [32]. The inclusion of rVV10 tends to lower the boundary position by $\approx 150 \mathrm{~K}$, with little to no effect outside $100 \leqslant$ $P \leqslant 200 \mathrm{GPa}$. The relative shift is significantly smaller than the difference between any two XC functionals [13,20-23]. That suggests deficiencies in the description of the short- and intermediate-range interactions across a wide range of $P T$ space as the cause for the variety of predictions. Fourth, in the low- $P$, high- $T$ regime there is evidence of a convergence of the SCAN-L+rVV10 and PBE [19] IMT boundaries that also is supported by the calculated optical and structural properties (see Supplemental Material [56]). This suggests that above such conditions, the use of PBE will provide about the same accuracy as SCAN.

\section{SUMMARY}

In summary, we have determined the IMT boundary of warm dense fluid hydrogen with consistent use of what is arguably the best approximate XC functional currently available for treating both molecular and condensed phase systems even handedly. The resulting hydrogen IMT boundary is in good agreement with experimental measurements across a wide range of pressures and temperatures. An analysis of the optical and structural properties shows concurrent, abrupt changes at the onset of a minimum metallic behavior. This signals a metallic transition due to an abrupt energy gap closure associated with the dissociation of molecular to atomic hydrogen. Furthermore, the inclusion of NQEs produces an explicit isotope effect in the form of clear splitting in the hydrogen and deuterium IMT boundaries.

\section{ACKNOWLEDGMENTS}

This work was supported by the Department of Energy National Nuclear Security Administration Award No. DENA0003856 and U.S. National Science Foundation PHY Grant No. 1802964. D.M.-R. and S.B.T. acknowledge support by U.S. Dept. of Energy Grant No. DE-SC 0002139. All computations were performed on the LLE HPC systems. This report was prepared as an account of work sponsored by an agency of the U.S. Government. Neither the U.S. Government nor any agency thereof, nor any of their employees, makes any warranty, express or implied, or assumes any legal liability or responsibility for the accuracy, completeness, or usefulness of any information, apparatus, product, or process disclosed, or represents that its use would not infringe 
privately owned rights. Reference herein to any specific commercial product, process, or service by trade name, trademark, manufacturer, or otherwise does not necessarily constitute or imply its endorsement, recommendation, or favoring by the U.S. Government or any agency thereof. The views and opinions of authors expressed herein do not necessarily state or reflect those of the U.S. Government or any agency thereof.
[1] G. Mazzola, R. Helled, and S. Sorella, Phase Diagram of Hydrogen and a Hydrogen-Helium Mixture at Planetary Conditions by Quantum Monte Carlo Simulations, Phys. Rev. Lett. 120, 025701 (2018).

[2] W. Lorenzen, B. Holst, and R. Redmer, Metallization in hydrogen-helium mixtures, Phys. Rev. B 84, 235109 (2011).

[3] J. E. P. Connerney, M. Benn et al., The Juno magnetic field investigation, Space Sci. Rev. 213, 39 (2017).

[4] J. M. McMahon, M. A. Morales, C. Pierleoni, and D. M. Ceperley, The properties of hydrogen and helium under extreme conditions, Rev. Mod. Phys. 84, 1607 (2012).

[5] S. X. Hu, V. N. Goncharov, T. R. Boehly, R. L. McCroy, S. Skupsky, L. A. Collins, J. D. Kress, and B. Militzer, Impact of first-principles properties of deuterium-tritium on inertial confinement fusion target designs, Phys. Plasmas 22, 056304 (2015).

[6] S. T. Weir, A. C. Mitchell, and W. J. Nellis, Metallization of Fluid Molecular Hydrogen at $140 \mathrm{GPa}$ (1.4 Mbar), Phys. Rev. Lett. 76, 1860 (1996).

[7] K. Ohta, K. Ichimaru, M. Einago, S. Kawaguchi, K. Shimizu, T. Matsuoka, N. Hirao, and Y. Ohishi, Phase boundary of hot dense fluid hydrogen, Sci. Rep. 5, 16560 (2015).

[8] M. Zaghoo, A. Salamat, and I. F. Silvera, Evidence of a firstorder phase transition to metallic hydrogen, Phys. Rev. B 93, 155128 (2016).

[9] V. Dzyabura, M. Zaghoo, and I. F. Silvera, Evidence of a liquidliquid phase transition in hot dense hydrogen, Proc. Natl. Acad. Sci. USA 110, 8040 (2013).

[10] R. S. McWilliams, D. Allen Dalton, M. F. Mahood, and A. F Goncharov, Optical Properties of Fluid Hydrogen at the Transition to a Conducting State, Phys. Rev. Lett. 116, 255501 (2016).

[11] M. Zaghoo and I. F. Silvera, Conductivity and dissociation in liquid metallic hydrogen and implications for planetary interiors, Proc. Natl. Acad. Sci. USA 114, 11873 (2017).

[12] M. Zaghoo, R. J. Husband, and I. F. Silvera, Striking isotope effect on the metallization phase lines of liquid hydrogen and deuterium, Phys. Rev. B 98, 104102 (2018).

[13] M. D. Knudson, M. P. Desjarlais, A. Becker, R. W. Lemke, K. R. Cochrane, M. E. Savage, D. E. Bliss, T. R. Mattsson, and R. Redmer, Direct observation of an abrupt insulator-to-metal transition in dense liquid deuterium, Science 348, 1455 (2015).

[14] P. M. Celliers et al., Insulator-metal transition in dense fluid deuterium, Science 361, 677 (2018).

[15] M. P. Desjarlais, M. D. Knudson, and R. Redmer, Comment on "Insulator-metal transition in dense fluid deuterium", Science 363, eaaw0969 (2019).

[16] P. M. Celliers et al., Response to Comment on "Insulator-metal transition in dense fluid deuterium", Science 363, eaaw1970 (2019).

[17] M. P. Desjarlais, M. D. Knudson, and R. Redmer, Thermodynamics of the insulator-metal transition in dense liquid deuterium, Phys. Rev. B 101, 104101 (2020).
[18] E. Engel and R. M. Dreizler, Density Functional Theory - An Advanced Course (Springer, Berlin, 2011).

[19] K. Burke and L. O. Wagner, DFT in a nutshell, Int. J. Quantum Chem. 113, 96 (2013); Erratum: DFT in a nutshell, 113, 1601(E) (2013).

[20] M. A. Morales, C. Pierleoni, E. Schwegler, and D. M. Ceperley, Evidence for a first-order liquid-liquid transition in highpressure hydrogen from ab initio simulations, Proc. Natl. Acad. Sci. USA 107, 12799 (2010).

[21] W. Lorenzen, B. Holst, and R. Redmer, First-order liquid-liquid phase transition in dense hydrogen, Phys. Rev. B 82, 195107 (2010).

[22] B. Holst, R. Redmer, and M. P. Desjarlais, Thermophysical properties of warm dense hydrogen using quantum molecular dynamics simulations, Phys. Rev. B 77, 184201 (2008).

[23] H. Y. Geng, Q. Wu, M. Marques, and G. J. Ackland, Thermodynamic anomalies and three distinct liquid-liquid transitions in warm dense liquid hydrogen, Phys. Rev. B 100, 134109 (2019).

[24] J. Yang, Ch. L. Tian, F. Sh. Liu, L. C. Cai, H. K. Yuan, M. M. Zhong, and F. Xiao, A new evidence of first-order phase transition for hydrogen at $3000 \mathrm{~K}$, Europhys. Lett. 109, 36003 (2015).

[25] C. Tian, P. Liu, H. Yuan, H. Chen, and A. Kuan, First-order liquid-liquid phase transition in compressed hydrogen and critical point, J. Chem. Phys. 150, 204114 (2019).

[26] J. P. Perdew, K. Burke, and M. Ernzerhof, Generalized Gradient Approximation Made Simple, Phys. Rev. Lett. 77, 3865 (1996); Generalized Gradient Approximation Made Simple [Phys. Rev. Lett. 77, 3865 (1996)], 78, 1396(E) (1996).

[27] V. V. Karasiev, J. W. Dufty, and S. B. Trickey, Nonempirical Semi-Local Free-Energy Density Functional for Matter under Extreme Conditions, Phys. Rev. Lett. 120, 076401 (2018).

[28] V. V. Karasiev, S. X. Hu, M. Zaghoo, and T. R. Boehly, Exchange-correlation thermal effects in shocked deuterium: Softening the principal Hugoniot and thermophysical properties, Phys. Rev. B 99, 214110 (2019).

[29] M. A. Morales, J. M. McMahon, C. Pierleoni, and D. M. Ceperley, Nuclear Quantum Effects and Nonlocal ExchangeCorrelation Functionals Applied to Liquid Hydrogen at High Pressure, Phys. Rev. Lett. 110, 065702 (2013).

[30] M. Dion, H. Rydberg, E. Schröder, D. C. Langreth, and B. I. Lundqvist, Van der Waals Density Functional for General Geometries, Phys. Rev. Lett. 92, 246401 (2004); Erratum: Van der Waals Density Functional for General Geometries [Phys. Rev. Lett. 92, 246401 (2004)], 95, 109902(E) (2005).

[31] K. Lee, É. D. Murray, L. Kong, B. I. Lundqvist, and D. C. Langreth, Higher-accuracy van der Waals density functional, Phys. Rev. B 82, 081101(R) (2010).

[32] B. Lu, D. Kang, D. Wang, T. Gao, and J. Dai, Towards the same line of liquid-liquid phase transition of dense hydrogen from various theoretical predictions, Chin. Phys. Lett. 36, 103102 (2019). 
[33] C. Pierleoni, M. A. Morales, G. Rillo, M. Holzmann, and D. M. Ceperley, Liquid-liquid phase transition in hydrogen by coupled electron-ion Monte Carlo simulations, Proc. Natl. Acad. Sci. USA 113, 4953 (2016).

[34] G. Rillo, M. A. Morales, D. M. Ceperley, and C. Pierleoni, Optical properties of high-pressure fluid hydrogen across molecular dissociation, Proc. Natl. Acad. Sci. USA 116, 9770 (2019).

[35] M. A. Morales, C. Pierleoni, and D. M. Ceperley, Equation of state of metallic hydrogen from coupled electron-ion Monte Carlo simulations, Phys. Rev. E 81, 021202 (2010).

[36] J. Heyd, G. E. Scuseria, and M. Ernzerhof, Hybrid functionals based on a screened Coulomb potential, J. Chem. Phys. 118, 8207 (2003); Erratum: "Hybrid functionals based on a screened Coulomb potential" [J. Chem. Phys. 118, 8207 (2003)], 124, 219906 (2003).

[37] R. Kubo, Statistical-mechanical theory of irreversible processes. I. General theory and simple applications to magnetic and conduction problems, J. Phys. Soc. Jpn. 12, 570 (1957).

[38] D. A. Greenwood, The Boltzmann equation in the theory of electrical conduction in metals, Proc. Phys. Soc. London 71, 585 (1958).

[39] J. Sun, A. Ruzsinszky, and J. P. Perdew, Strongly Constrained and Appropriately Normed Semilocal Density Functional, Phys. Rev. Lett. 115, 036402 (2015).

[40] O. A. Vydrov and T. Van Voorhis, Nonlocal van der Waals density functional: The simpler the better, J. Chem. Phys. 133, 244103 (2010).

[41] R. Sabatini, T. Gorni, and S. de Gironcoli, Nonlocal van der Waals density functional made simple and efficient, Phys. Rev. B 87, 041108 (2013).

[42] H. Peng, Z.-H. Yang, J. P. Perdew, and J. Sun, Versatile van Der Waals Density Functional Based on a Meta-Generalized Gradient Approximation, Phys. Rev. X 6, 041005 (2016).

[43] G. Kresse and J. Hafner, Ab initio molecular-dynamics simulation of the liquid-metal-amorphous-semiconductor transition in germanium, Phys. Rev. B 49, 14251 (1994).

[44] G. Kresse and J. Furthmüller, Efficiency of ab-initio total energy calculations for metals and semiconductors using a plane-wave basis set, Comput. Mater. Sci. 6, 15 (1996).

[45] G. Kresse and J. Furthmüller, Efficient iterative schemes for $a b$ initio total-energy calculations using a plane-wave basis set, Phys. Rev. B 54, 11169 (1996).

[46] G. Kresse and D. Joubert, From ultrasoft pseudopotentials to the projector augmented-wave method, Phys. Rev. B 59, 1758 (1999).

[47] D. Marx and M. Parrinello, Ab initio path integral molecular dynamics: Basic ideas, J. Chem. Phys. 104, 4077 (1996).

[48] M. Ceriotti, J. More, and D. E. Manolopoulos, i-PI: A python interface for ab initio path integral molecular dynamics simulations, Comput. Phys. Commun. 185, 1019 (2014).
[49] V. Kapil et al., i-PI 2.0: A universal force engine for advanced molecular simulations, Comput. Phys. Commun. 236, 214 (2019)

[50] P. Giannozzi et al., Quantum Espresso: A modular and open-source software project for quantum simulations of materials, J. Phys.: Condens. Matter 21, 395502 (2009).

[51] P. Giannozzi et al., Advanced capabilities for materials modelling with quantum espresso, J. Phys.: Condens. Matter 29, 465901 (2017).

[52] V. V. Karasiev, T. Sjostrom, and S. B. Trickey, Generalizedgradient approximation noninteracting free energy functionals for orbital free density functional calculations, Phys. Rev. B 86, 115101 (2012).

[53] D. Mejía-Rodríguez and S. B. Trickey, Deorbitalization strategies for meta-generalized-gradient-approximation exchange-correlation functionals, Phys. Rev. A 96, 052512 (2017).

[54] D. Mejía-Rodríguez and S. B. Trickey, Deorbitalized metaGGA exchange-correlation functionals in solids, Phys. Rev. B 98, 115161 (2018).

[55] L. Calderín, V. V. Karasiev, and S. B. Trickey, KuboGreenwood electrical conductivity formulation and implementation for projector augmented wave datasets, Comput. Phys. Commun. 221, 118 (2017).

[56] See Supplemental Material at http://link.aps.org/supplemental/ 10.1103/PhysRevResearch.2.032065 for more technical details of BOMD, PIMD, KG conductivity and reflectivity, and vibrational-rotational autocorrelation calculations, convergence studies and sampling tests, PCF testing, and dc conductivity testing, the comparison of SCAN and SCAN-L, the comparison with PBE and vdW-DF1 and for the vibrational/rotational analysis.

[57] V. Gorelov, C. Pierleoni, and D. M. Ceperley, Benchmarking vdW-DF first-principles predictions against coupled electronion Monte Carlo for high-pressure liquid hydrogen, Contrib. Plasma Phys. 59, e201800185 (2019).

[58] P. Davis et al., X-ray scattering measurements of dissociationinduced metallization of dynamically compressed deuterium, Nat. Commun. 7, 11189 (2016).

[59] J. P. Perdew and A. Zunger, Self-interaction correction to density-functional approximations for many-electron systems, Phys. Rev. B 23, 5048 (1981).

[60] A. V. Krukau, O. A. Vydrov, A. F. Izmaylov, and G. E. Scuseria, Influence of the exchange screening parameter on the performance of screened hybrid functionals, J. Chem. Phys. 125, 224106 (2006).

[61] C. S. Zha, H. Liu, J. S. Tse, and R. J. Hemley, Melting and High P-T Transitions of Hydrogen up to $300 \mathrm{GPa}$, Phys. Rev. Lett. 119, 075302 (2017). 